\title{
First Principles DFT Study of Hydrogen Storage on Graphene with La Decoration
}

\author{
Yuanyuan Li, Yiming Mi*, Gaili Sun \\ School of Chemistry and Chemical Engineering, Shanghai University of Engineering Science, Shanghai, China \\ Email: "yimingmi@sues.edu.cn
}

Received 30 November 2015; accepted 26 December 2015; published 29 December 2015

Copyright ( 2015 by authors and Scientific Research Publishing Inc.

This work is licensed under the Creative Commons Attribution International License (CC BY).

http://creativecommons.org/licenses/by/4.0/

(c) (i) Open Access

\begin{abstract}
The properties of hydrogen storage on graphene with La decoration are investigated using a firstprinciples plane-wave pseudopotential method based on the density functional theory in this paper. The clustering problem of La decorated graphene is considered and B doping can solve it effectively in theory. We obtain the stable geometrical configuration of the modified system and the properties of hydrogen storage are excellent. It can absorb up to $6 \mathrm{H}_{2}$ molecules with an average adsorption energy range of -0.529 to $-0.655 \mathrm{eV} / \mathrm{H}_{2}$, which meets the ideal range between the physisorbed and chemisorbed states for hydrogen storage. Furthermore, it is proved that the existence of La atom alters the charge distribution of $\mathrm{H}_{2}$ molecules and graphene sheet based on the calculation and analysis about the electronic density of states and charge density difference of the modified system. La atom interacts with hydrogen molecules through Kubas interaction. Thereby, it improves the performance of graphene sheet for hydrogen storage. The modified system exhibits the excellent potential to become one of the most suitable candidates for hydrogen storage medium at near ambient conditions with molecule state.
\end{abstract}

\section{Keywords}

Graphene, La Decoration, First-Principles, Hydrogen Storage

\section{Introduction}

Due to the high energy density and clean fuel source, hydrogen as a promising alternative energy has attracted great attention all over the world [1] [2]. However, there is a major challenge to find an excellent hydrogen storage medium under normal conditions [3] [4]. The desirable system for storing hydrogen should have high gra-

*Corresponding author.

How to cite this paper: Li, Y.Y., Mi, Y.M. and Sun, G.L. (2015) First Principles DFT Study of Hydrogen Storage on Graphene with La Decoration. Journal of Materials Science and Chemical Engineering, 3, 87-94.

http://dx.doi.org/10.4236/msce.2015.312013 
vimetric and volumetric densities at near room temperature and ambient pressure [5]. Moreover, the US Department of Energy (DOE) has set a target for the efficient materials to store hydrogen, which requires gravimetric and volumetric densities of $7.5 \mathrm{wt} \%$ and $70 \mathrm{~g} / \mathrm{L}$, respectively, an operating temperature between $-40^{\circ} \mathrm{C}$ and $60^{\circ} \mathrm{C}$ and the safe, durable (1500 operational cycle life) storage system [6] [7]. In addition, the ideal adsorption energy should be in the range of $-0.2--0.7 \mathrm{eV} / \mathrm{H}_{2}$ [8]. This range of energy is intermediate between the physisorbed and chemisorbed states. It is ideal for hydrogen storage under ambient pressure and temperature. To achieve this, a variety of materials about hydrogen storage have been investigated by several theoretical and experimental studies. The development of metal hydride starts early [9] [10] and other materials begin to be developed rapidly. But it is still an open-ended problem to find suitable novel materials for hydrogen storage which can meet the requirements.

In recent years, carbon nanomaterials have been widely studied for hydrogen storage [11]-[13]. Due to the light weight, high specific surface and unique properties, graphene has become a promising material to store hydrogen [14]. However, similar to other carbon nanomaterials, the interaction between graphene and $\mathrm{H}_{2}$ is through weak van der Waals forces with low adsorption energy $\sim-0.1 \mathrm{eV} / \mathrm{H}_{2}$ at ambient conditions [15]-[17]. Thus, it is meaningless for using pristine graphene to store hydrogen. Recently, to improve the binding energy between graphene and $\mathrm{H}_{2}$ molecules, many studies have been devoted to modifying the surface of graphene with transition metals (TMs) [18]-[21]. The TM atom interacts with $\mathrm{H}_{2}$ molecules through Kubas [22] interactions due to the empty $d$ orbitals. It increases the binding ability of hydrogen with graphene. However, because of the high cohesive energy, TMs tend to form cluster on the surface of the carbon nanostructures easily [23] [24]. My understanding is that, typically, TM-TM is much stronger than grapheme-TM. Therefore, it is difficult to achieve uniformly coated monolayer of TM atoms experimentally. This is unfavorable for the capacity of hydrogen storage.

Numerous studies have shown that, boron (B) doped carbon nanomaterials can prevent the TMs clustering availably. B acts as a strong charge acceptor and consequently enhances the adsorption energy of foreign atoms on the surface of the carbon nanostructures due to its empty $p$ orbital [25]. Nachimuthu et al. [26] investigated that TM atoms such as the Ni, Pd and Co atoms are suitable for decorating B-doped graphene surface, which can be adsorbed stably on the surface of graphene. Besides, B can be easily incorporated substitutionally in the hexagonal structure of graphene and change the electronic structure of graphene effectively to prevent the clustering of foreign atoms. It has been proved that B doped graphene has been successfully synthesized experimentally [27] [28].

Because of the chemical activity and small cohesive energy, the La-Ni alloy has been used as hydrogen storage materials; La has great potential in the aspect of storing hydrogen. We focus primarily on the stable geometrical configuration of graphene with La decoration, the adsorption of hydrogen and the effects of La for the process of hydrogen storage using a first-principles plane-wave pseudopotential simulations based on the density functional theory in this paper.

\section{Simulation Details}

All the density-functional theory (DFT) calculations are performed using a plane-wave basis set with the projector augmented plane wave (PAW) method as implemented in the Vienna ab initio simulation package (VASP) [29]-[31]. We select the local-density approximation (LDA) as the exchange and correlation potential for our work. Although no DFT functional describes accurately all the characteristics of molecular interactions, especially van der Waals (vdW) interactions [32]-[34], the prediction of the physisorption energies of $\mathrm{H}_{2}$ on the surface of carbon nanomaterials as based on the LDA are in good agreement with experiments [35]-[37]. More than that, the overestimate of the binding energy by LDA is almost compensated by the ignored van der Waals interactions [38] [39]. The electron wave functions are expanded by plane waves with a kinetic energy cutoff of 450 $\mathrm{eV}$ to attain the required convergence. All of the self-consistent loops are iterated until the total energy difference of the systems between the adjacent iterating steps is less than $10^{-7} \mathrm{eV}$. The Brillouin zone is sampled by 5 $\times 5 \times 1$ mesh points in k-space based on Monkhorst-Pack scheme [40]. The effective range of the kinetic energy cutoff and the validity of the mesh density used in this calculation are determined by a convergence test using the theoretically estimated lattice constants of the pristine graphene, $2.46 \AA$. To avoid the interactions of adjacent slabs, the vacuum space of $20 \AA$ is introduced for $4 \times 4$ supercell which contains 32 carbon atoms. 


\section{Results and Discussions}

\subsection{The Decoration of La}

Initially, we assume that La atoms distributed uniformly on graphene sheet and study the adsorption behavior of $\mathrm{La}$ on pristine graphene. The adsorption energy of La on graphene is defined as

$$
E_{\text {ad-La }}=E_{\mathrm{La} / \text { graphene }}-E_{\mathrm{La}}-E_{\text {graphene }}
$$

where $E_{\mathrm{La} / \text { graphene }}, E_{\mathrm{La}}$, and $E_{\text {graphene }}$ are the total energies of the La-decorated graphene sheet, an isolated La atom, and pristine graphene sheet, respectively. Similarto other rare earth metal atoms attaching on carbon nanomaterials [41] [42], the favorite adsorption site for an isolated La atom on the surface of graphene is the center of a hexagonal ring. Meanwhile, the adsorption energy of La on grapheme $E_{\text {ad-La }}$ is $-2.99 \mathrm{eV}$ which is calculated by Formula (1). However, the cohesive energy of bulk solid phase La $(-4.47 \mathrm{eV} / \mathrm{atom})$ is higher than the calculated adsorption energy of La atom on pristine graphene. It indicates that La atoms tend to cluster.

In order to have a stable and uniform decoration of individual $\mathrm{La}$ atoms on graphene, we introduce $\mathrm{B}$ atom to $4 \times 4 \times 1$ graphene supercell. The adsorption energy of La on B-doped graphene is calculated by

$$
E_{\text {ad-La }}=E_{\mathrm{La} / \mathrm{B} / \text { graphene }}-E_{\mathrm{La}}-E_{\mathrm{B} / \text { graphene }}
$$

where $E_{\mathrm{L} a / \mathrm{B} / \text { graphene }}, E_{\mathrm{La}}$, and $E_{\mathrm{B} / \text { graphene }}$ are the total energies of the La decorated B doped graphene sheet, an isolated $\mathrm{La}$ atom, and $\mathrm{B}$ doped graphene sheet, respectively.

We obtain the stable configuration after the modified system is fully relaxed. As shown in Figure 1, the La atom tends to stay near the top site above the B atom and the distance between $\mathrm{La}$ and $\mathrm{B}$ atom is $2.43 \mathrm{~A}$. Simulation results show that the adsorption energy of $\mathrm{La}$ atom on B-doped graphene sheet is $-4.59 \mathrm{eV} /$ atom which is higher than the cohesive energy of bulk $\mathrm{La}(-4.47 \mathrm{eV} /$ atom). It turns that B doping can prevent the cluster of $\mathrm{La}$ atoms on graphene sheet successfully in theory.

\subsection{The Adsorption of Hydrogen on La/B/Graphene}

To explore the performance of the active structure La/B/Graphene for hydrogen storage, we investigate the behavior of $\mathrm{H}_{2}$ molecules adsorbed on La-decorated graphene. The finally optimized configurations after full relaxation of all $\mathrm{H}_{2}$ molecules are shown in Figures 2(a)-(f). The Figures 2(a)-(f) show that $\mathrm{H}_{2}$ molecules always take the sites near La atoms and the vertical distance between the first $\mathrm{H}_{2}$ molecule and graphene sheet is $2.50 \AA$, other $\mathrm{H}_{2}$ molecules have similar distance with graphene. It should be noticed that the average bond length $d_{\mathrm{H}-\mathrm{H}}$ is about $0.85 \AA$, which is elongated compared to the length $0.74 \AA$ in gas-phase $\mathrm{H}_{2}$ molecules. From all configurations we can also find that there is slight distortion especially in-plane distortion to the graphene as displayed in the side view of Figure 2. The similar phenomenon can be observed from the adsorption of other $3 d$-transition metals which induces lattice distortion in the graphene layer [43].

To examine the stability that $\mathrm{H}_{2}$ molecules adsorbed on the modified graphene, we calculate the average adsorption energy of $\mathrm{H}_{2}$ molecules by Formula (3)

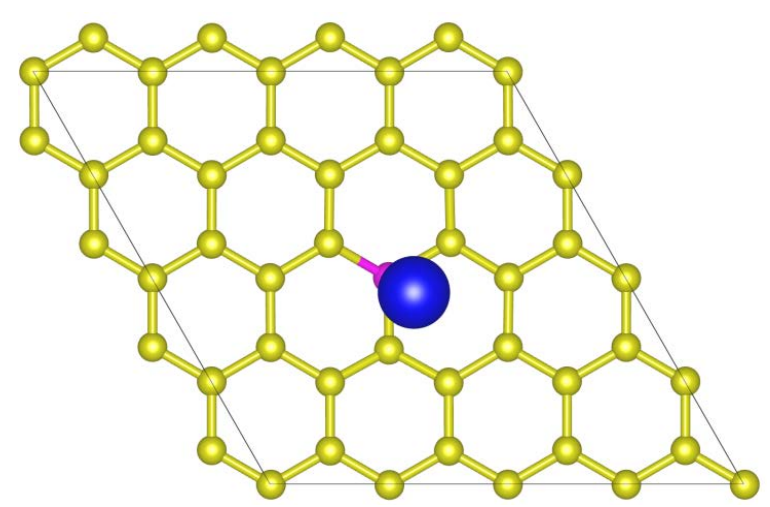

Figure 1. The stable structure of La decorated graphene sheet, where yellow, rose and gray balls stand for $\mathrm{C}$ atom, $\mathrm{B}$ atom and La atom, respectively. 


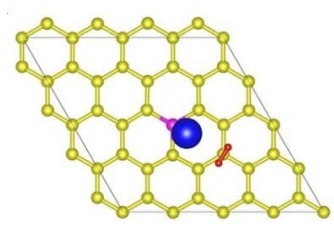

(a)

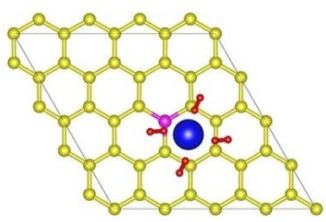

(d)

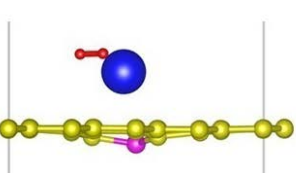

(a)

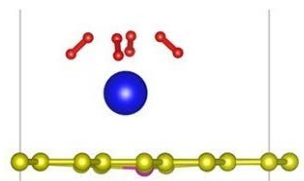

(d)

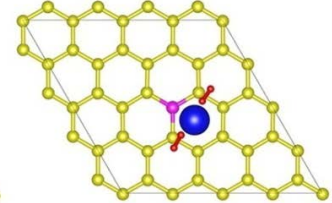

(b)

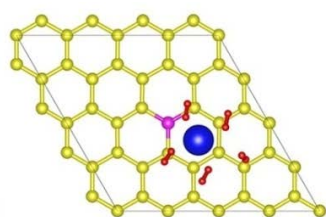

(e)

(I)

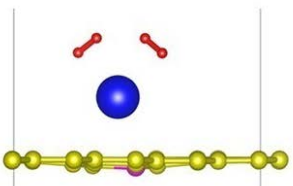

(b)

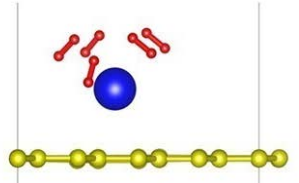

(e)

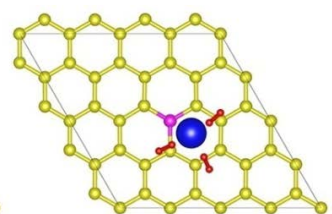

(c)

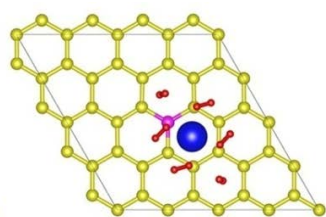

(f)

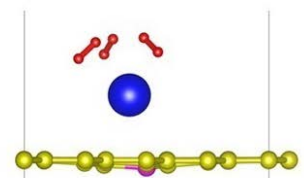

(c)

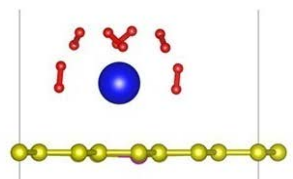

(f)

(II)

Figure 2. (a)-(f) depict the optimized structures of $\mathrm{H}_{2}$ molecules adsorption on $\mathrm{La} / \mathrm{B} /$ grapheme (I: top view, II: side view. Red balls stand for $\mathrm{H}$ atom).

$$
E_{\text {ad- } \mathrm{H}_{2}}=\left(E_{\text {total }}-E_{\mathrm{La} / \mathrm{B} / \text { graphene }}-n E_{\mathrm{H}_{2}}\right) / n
$$

where $E_{\text {total }}, E_{\mathrm{L} a / \mathrm{B} / \text { rraphene }}$ and $E_{\mathrm{H}_{2}}$ are the total energy of the system with $\mathrm{H}_{2}$ adsorbed on the modified graphene, La-decorated graphene, and a free $\mathrm{H}_{2}$ molecule, respectively. Where $n$ denotes the number of adsorbed $\mathrm{H}_{2}$. The average adsorption energy of $\mathrm{H}_{2}$ molecules are summarized in Table 1. From Table 1 we can see that the distances between La atom and graphene change a little. Moreover, the range of the average adsorption energy is from -0.529 to $-0.655 \mathrm{eV} / \mathrm{H}_{2}$ which can meet the ideal adsorption energy range $-0.2--0.7 \mathrm{eV} / \mathrm{H}_{2}$ between the physisorbed and chemisorbed states. Thereby, all $\mathrm{H}_{2}$ molecules absorbed on the modified system can be controlled under normal conditions. However, when seven $\mathrm{H}_{2}$ molecules are close to the active system, the system is unable to provide more energy to adsorb these molecules. Thus, we infer that the maximum number of $\mathrm{H}_{2}$ molecules adsorbed on the $4 \times 4$ modified graphene supercell is 7 . In conclusion, the B-doped graphene with La decoration has an excellent performance for hydrogen storage under ambient conditions.

\subsection{The Influence of La on Hydrogen Storage}

In order to get a better understanding about the influence of La atom on the process of hydrogen storage, we analyze the interaction between La atom with graphene and $\mathrm{H}_{2}$ molecules. B atom can accept charge from $\mathrm{C}$ atoms and enhance the adsorption energy of La atom on the surface of graphene due to its empty $p$ orbitals. Then, the La atom can be adsorbed stably. Furthermore, we analyze the total and partial density of states for the system $1 \mathrm{H}_{2} / \mathrm{La} / \mathrm{B} / \mathrm{Graphene}$ as displayed in Figure 3 . It clearly shows that there are several overlaps of main peaks of $s$ orbitals of $\mathrm{H}_{2}$ molecule and the $d$ orbitals of $\mathrm{La}$ atom at around $-0.1 \mathrm{eV}$, indicating strong binding and hybridizing between orbitals of $\mathrm{H}_{2}$ molecule and La. It can be inferred that there is a small charge transfer from the 


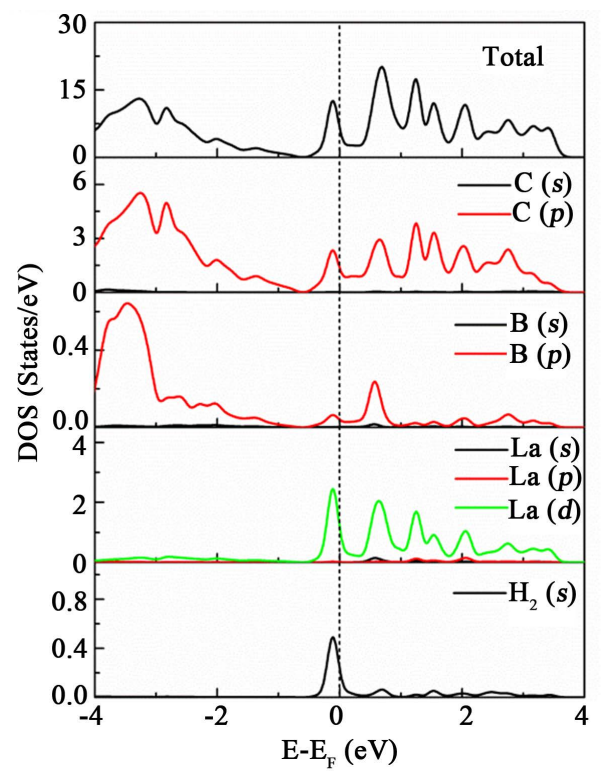

Figure 3. The total and partial density of states of $1 \mathrm{H}_{2} / \mathrm{La} / \mathrm{B} /$ graphene system.

Table 1. The average adsorption energy of $\mathrm{H}_{2}$ molecules and the distances between La atom and graphene sheet.

\begin{tabular}{ccc}
\hline No. of $\mathrm{H}_{2}$ molecules & $E_{\text {ad- } \mathrm{H}_{2}}\left(\mathrm{eV} / \mathrm{H}_{2}\right)$ & $d_{\text {La } \perp \mathrm{G}}(\AA)$ \\
\hline $1 \mathrm{H}_{2}$ & -0.655 & 1.856 \\
$2 \mathrm{H}_{2}$ & -0.565 & 2.275 \\
$3 \mathrm{H}_{2}$ & -0.576 & 2.386 \\
$4 \mathrm{H}_{2}$ & -0.599 & 2.206 \\
$5 \mathrm{H}_{2}$ & -0.577 & 1.978 \\
$6 \mathrm{H}_{2}$ & -0.529 & 2.301 \\
\hline
\end{tabular}

$\sigma$-bonding orbitals of $\mathrm{H}_{2}$ molecule to the empty $d$ orbitals of La atom. While the back donation from the filled states of $d$ to the $\sigma^{*}$-antibonding orbitals of $\mathrm{H}_{2}$ molecule enhances the interaction. It is a typical Kubas interaction [44] [45]. This charge transfer is responsible for the elongation of $\mathrm{H}-\mathrm{H}$ bond length in $\mathrm{H}_{2}$ molecules and improves the adsorption ability between La atom and graphene sheet.

Meanwhile, to illustrate the electronic distribution of system, we calculate the charge density difference of the system caused by one $\mathrm{H}_{2}$ molecule which is determined by the following function

$$
\Delta \rho=\rho_{\mathrm{G} / \mathrm{B} / \mathrm{La}-\mathrm{H}_{2}}-\rho_{\mathrm{G} / \mathrm{B} / \mathrm{La}}-\rho_{\mathrm{H}_{2}}
$$

where $\rho_{\mathrm{G} / \mathrm{B} / \mathrm{La}-\mathrm{H}_{2}}, \quad \rho_{\mathrm{G} / \mathrm{B} / \mathrm{La}}$ and $\rho_{\mathrm{H}_{2}}$ are the distribution of charge density difference about adsorbing surface, the sheet and isolated $\mathrm{H}_{2}$ molecule, respectively. The corresponding distribution of charge density difference is shown in Figure 4. It clearly shows that on the surface of sheet, the electron density increases in the region between $\mathrm{H}_{2}$ molecule and $\mathrm{La}$ atom and this causes the electron density of surrounding $\mathrm{C}$ atoms to decrease. This means that $\mathrm{H}_{2}$ molecule and La atom interact with each other, and there is also interaction between graphene sheet and La. The results are consistent with the analysis of the total and partial density of states. In conclusion, La atom plays an important role in the process of hydrogen storage.

\section{Conclusion}

We have performed first-principles electronic structure calculations to study the properties of hydrogen storage on graphene with La decoration. A stable and uniform decoration of individual La atom on graphene can be ob- 


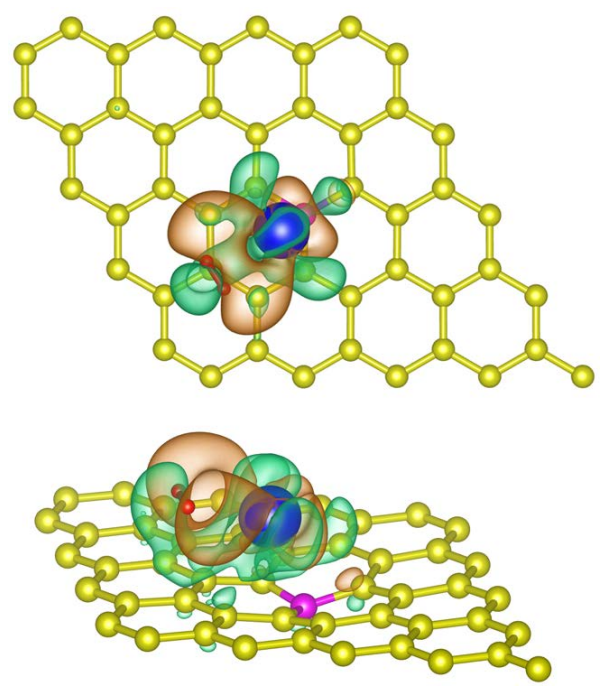

Figure 4. The electron charge density difference for $1 \mathrm{H}_{2} / \mathrm{La} / \mathrm{B} /$ graphene system, where orange and green contours stand for high and low electron density region, respectively.

tained upon substitutional B doping. We find the stable geometry configurations of $\mathrm{H}_{2}$ molecules absorbed on graphene sheet with La decoration. The modified system can absorb $6 \mathrm{H}_{2}$ molecules with the adsorption energy rang from -0.529 to $-0.655 \mathrm{eV} / \mathrm{H}_{2}$ which meets the ideal adsorption energy range for $\mathrm{H}_{2}$ molecules to be recycled at near ambient conditions. The calculation and analysis of electronic structure demonstrate that $\mathrm{La}$ atom interacts with $\mathrm{H}_{2}$ molecules and graphene through the charge-transfer. La atom becomes a bridge linking $\mathrm{H}_{2}$ molecules and graphene and improves the adsorption capacity of graphene sheet for hydrogen storage. Thereby, the modified system displays the outstanding potential to become one of suitably promising candidate materials for hydrogen storage.

\section{Acknowledgements}

This work was supported by Innovation Program of Shanghai Municipal Education Commission, China (10YZ172) and Subjects Construction Program of Shanghai University of Engineering Science, China (2012gp43).

\section{References}

[1] Schlapbach, L. and Züttel, A. (2001) Hydrogen-Storage Materials for Mobile Applications. Nature, 414, 353-358. http://dx.doi.org/10.1038/35104634

[2] Tollefson, J. (2010) Hydrogen Vehicles: Fuel of the Future. Nature, 464, 1262-1264. http://dx.doi.org/10.1038/4641262a

[3] Marban, G. and Vales-Solis, T. (2007) Towards the Hydrogen Economy? International Journal of Hydrogen Energy, 32, 1625-1637. http://dx.doi.org/10.1016/j.ijhydene.2006.12.017

[4] Miwa, R.H., Martins, T.B. and Fazzio, A. (2008) Hydrogen Adsorption on Boron Doped Graphene: An ab initio Study. Nanotechnology, 19, Article ID: 155708. http://dx.doi.org/10.1088/0957-4484/19/15/155708

[5] Jena, P. (2011) Materials for Hydrogen Storage: Past, Present, and Future. Journal of Physical Chemistry Letters, 2, 206-211. http://dx.doi.org/10.1021/jz1015372

[6] US Department of Energy’s Energy Efficiency and Renewable Energy Website. http://www.hydrogen.energy.gov/annual_progress14_storage.html\#c

[7] Yoon, M., Yang, S., Hicke, C., Wang, E., Geohegan, D. and Zhang, Z. (2008) Calcium as the Superior Coating Metal in Functionalization of Carbon Fullerenes for High-Capacity Hydrogen Storage. Physical Review Letters, 100, Article ID: 206806. http://dx.doi.org/10.1103/physrevlett.100.206806

[8] Ao, Z., Dou, S., Xu, Z., Jiang, Q. and Wang, G. (2014) Hydrogen Storage in Porous Graphene with Al Decoration. International Journal of Hydrogen Energy, 39, 16244-16251. http://dx.doi.org/10.1016/j.ijhydene.2014.01.044

[9] Schuth, F., Bogdanovic, B. and Felderhoff, M. (2004) Light Metal Hydrides and Complex Hydrides for Hydrogen Sto- 
rage. Chemical Communications, 20, 2249-2258. http://dx.doi.org/10.1039/b406522k

[10] Fukuzumi, S. and Suenobu, T. (2013) Hydrogen Storage and Evolution Catalysed by Metal Hydride Complexes. Dalton Transactions, 42, 18-28. http://dx.doi.org/10.1039/C2DT31823G

[11] Yildirim, T. and Ciraci, S. (2005) Titanium-Decorated Carbon Nanotubes as a Potential High-Capacity Hydrogen Storage Medium. Physical Review Letters, 94, Article ID: 175501. http://dx.doi.org/10.1103/PhysRevLett.94.175501

[12] Shalabi, A.S., Taha, H.O., Soliman, K.A. and Abeld, A.S. (2014) Hydrogen Storage Reactions on Titanium Decorated Carbon Nanocones Theoretical Study. Journal of Power Sources, 271, 32-41. http://dx.doi.org/10.1016/j.jpowsour.2014.07.158

[13] Ataca, C., Aktürk, E. and Ciraci, S. (2009) Hydrogen Storage of Calcium Atoms Adsorbed on Graphene: First-Principles Plane Wave Calculations. Physical Review B, 79, Article ID: 041406. http://dx.doi.org/10.1103/PhysRevB.79.041406

[14] Stankovich, S., Dikin, D.A., Dommett, G.H.B, Kohlhaas, K.M., Zimney, E.J., Stach, E.A., Piner, R.D., Nguyen, S.T. and Ruoff, R.S. (2006) Graphene-Based Composite Materials. Nature, 442, 282-286. http://dx.doi.org/10.1038/nature04969

[15] Panella, B., Hirscher, M. and Roth, S. (2005) Hydrogen Adsorption in Different Carbon Nanostructures. Carbon, 43, 2209-2214. http://dx.doi.org/10.1016/j.carbon.2005.03.037

[16] Ritschel, M., Uhlemann, M., Gutfleisch, O., Leonhardt, A., Graff, A., Täschner, C. and Fink, J. (2002) Hydrogen Storage in Different Carbon Nanostructures. Applied Physics Letters, 80, 2985-2987. http://dx.doi.org/10.1063/1.1469680

[17] Patchkovskii, S., Tse, J.S., Yurchenko, S.N., Zhechkov, L., Heine, T. and Seifert, G. (2005) Graphene Nanostructures as Tunable Storage Media for Molecular Hydrogen. Proceedings of the National Academy of Sciences of the United States of America, 102, 10439-10444. http://dx.doi.org/10.1073/pnas.0501030102

[18] Bhattacharya, A., Bhattacharya, S., Majumder, C. and Das, G. (2010) Transition-Metal Decoration Enhanced RoomTemperature Hydrogen Storage in a Defect-Modulated Graphene Sheet. Journal of Physical Chemistry C, 114, 1029710301. http://dx.doi.org/10.1021/jp100230c

[19] Chu, S., Hu, L., Hu, X., Yang, M. and Deng, J. (2011) Titanium-Embedded Graphene as High-Capacity HydrogenStorage Media. International Journal of Hydrogen Energy, 36, 12324-12328. http://dx.doi.org/10.1016/j.ijhydene.2011.07.015

[20] Gaboardi, M., Bliersbach, A., Bertoni, G., Aramini, M., Vlahopoulou, G., Pontiroli, D., Mauron, P., Magnani, G., Salviati, G. and Züttel, A. (2014) Decoration of Graphene with Nickel Nanoparticles: Study of the Interaction with Hydrogen. Journal of Materials Chemistry A, 2, 1039-1046. http://dx.doi.org/10.1039/C3TA14127F

[21] López-Corral, I., Germán, E.A., Juan, A., Volpe, M.A.A. and Brizuela, G.P. (2011) DFT Study of Hydrogen Adsorption on Palladium Decorated Graphene. Journal of Physical Chemistry C, 115, 4315-4323. http://dx.doi.org/10.1021/jp110067w

[22] Kubas, G.J. (1988) Molecular Hydrogen Complexes: Coordination of a $\sigma$ Bond to Transition Metals. Accounts of Chemical Research, 21, 120-128. http://dx.doi.org/10.1021/ar00147a005

[23] Durgun, E., Ciraci, S., Zhou, W. and Yildirim, T. (2006) Transition-Metal-Ethylene Complexes as High-Capacity Hydrogen-Storage Media. Physical Review Letters, 97, Article ID: 226102. http://dx.doi.org/10.1103/PhysRevLett.97.226102

[24] Sun, Q., Wang, Q., Jena, P. and Kawazoe, Y. (2005) Clustering of Ti on a $C_{60}$ Surface and Its Effect on Hydrogen Storage. Journal of the American Chemical Society, 127, 14582-14583. http://dx.doi.org/10.1021/ja0550125

[25] Beheshti, E., Nojeh, A. and Servati, P. (2011) A First-Principles Study of Calcium-Decorated, Boron-Doped Graphene for High Capacity Hydrogen Storage. Carbon, 49, 1561-1567. http://dx.doi.org/10.1016/j.carbon.2010.12.023

[26] Nachimuthu, S., Lai, P.J. and Jiang, J.C. (2014) Efficient Hydrogen Storage in Boron Doped Graphene Decorated by Transition Metals-A First-Principles Study. Carbon, 73, 132-140. http://dx.doi.org/10.1016/j.carbon.2014.02.048

[27] Shirasaki, T., Derré, A., Ménétrier, M., Tressaud, A. and Flandrois, S. (2000) Synthesis and Characterization of Boron-Substituted Carbons. Carbon, 38, 1461-1467. http://dx.doi.org/10.1016/S0008-6223(99)00279-1

[28] Chung, T.M., Jeong, Y., Chen, Q., Kleinhammes, A. and Wu, Y. (2008) Synthesis of Microporous Boron-Substituted Carbon (B/C) Materials Using Polymeric Precursors for Hydrogen Physisorption. Journal of the American Chemical Society, 130, 6668-6669. http://dx.doi.org/10.1021/ja800071y

[29] Kresse, G. and Hafner, J. (1993) Ab Initio Molecular Dynamics for Open-Shell Transition Metals. Physical Review B, 48, 13115-13118. http://dx.doi.org/10.1103/PhysRevB.48.13115

[30] Kresse, G. and Furthmüller, J. (1996) Efficiency of Ab-Initio Total Energy Calculations for Metals and Semiconductors Using a Plane-Wave Basis Set. Computational Materials Science, 6, 15-50. http://dx.doi.org/10.1016/0927-0256(96)00008-0 
[31] Kresse, G. and Furthmüller, J. (1996) Efficient Iterative Schemes for Ab initio Total-Energy Calculations Using a Plane-Wave Basis Set. Physical Review B, 54, 11169-11186. http://dx.doi.org/10.1103/PhysRevB.54.11169

[32] Cohen, A.J., Mori-Sánchez, P. and Yang, W. (2008) Insights into Current Limitations of Density Functional Theory. Science, 321, 792-794. http://dx.doi.org/10.1126/science.1158722

[33] Khantha, M., Cordero, N., Molina, L., Alonso, J. and Girifalco, L. (2004) Interaction of Lithium with Graphene: An Ab initio Study. Physical Review B, 70, Article ID: 125422. http://dx.doi.org/10.1103/PhysRevB.70.125422

[34] Lee, K., Murray, É.D., Kong, L., Lundqvist, B.I. and Langreth, D.C. (2010) Higher-Accuracy van der Waals Density Functional. Physical Review B, 82, Article ID: 081101. http://dx.doi.org/10.1103/PhysRevB.82.081101

[35] Perdew, J.P. and Zunger, A. (1981) Self-Interaction Correction to Density-Functional Approximations for Many-Electron Systems. Physical Review B, 23, 5048-5079. http://dx.doi.org/10.1103/PhysRevB.23.5048

[36] Okamoto, Y. and Miyamoto, Y. (2001) Ab initio Investigation of Physisorption of Molecular Hydrogen on Planar and Curved Graphenes. Journal of Physical Chemistry B, 105, 3470-3474. http://dx.doi.org/10.1021/jp003435h

[37] Ao, Z., Jiang, Q., Zhang, R., Tan, T. and Li, S. (2009) Al Doped Graphene: A Promising Material for Hydrogen Storage at Room Temperature. Journal of Applied Physics, 105, Article ID: 074307. http://dx.doi.org/10.1063/1.3103327

[38] Leenaerts, O., Partoens, B. and Peeters, F. (2008) Adsorption of $\mathrm{H}_{2} \mathrm{O}, \mathrm{NH}_{3}, \mathrm{CO}, \mathrm{NO}_{2}$, and $\mathrm{NO}$ on Graphene: A FirstPrinciples Study. Physical Review B, 77, Article ID: 125416. http://dx.doi.org/10.1103/PhysRevB.77.125416

[39] Cabria, I., López, M. and Alonso, J. (2008) Hydrogen Storage in Pure and Li-Doped Carbon Nanopores: Combined Effects of Concavity and Doping. Journal of Chemical Physics, 128, Article ID: 144704. http://dx.doi.org/10.1063/1.2900964

[40] Monkhorst, H.J. and Pack, J.D. (1976) Special Points for Brillouin-Zone Integrations. Physical Review B, 13, 51885192. http://dx.doi.org/10.1103/PhysRevB.13.5188

[41] Chakraborty, B., Modak, P. and Banerjee, S. (2012) Hydrogen Storage in Yttrium-Decorated Single Walled Carbon Nanotube. Journal of Physical Chemistry C, 116, 22502-22508. http://dx.doi.org/10.1021/jp3036296

[42] Fair, K.M., Cui, X.Y. and Li, L. (2013) Hydrogen Adsorption Capacity of Adatoms on Double Carbon Vacancies of Graphene: A Trend Study from First Principles. Physical Review B, 87, Article ID: 014102. http://dx.doi.org/10.1103/PhysRevB.87.014102

[43] Liu, X., Wang, C.Z., Yao, Y.X., Lu, W.C., Hupalo, M., Tringides, M.C. and Ho, K.M. (2011) Bonding and Charge Transfer by Metal Adatom Adsorption on Graphene. Physical Review B, 83, Article ID: 235411. http://dx.doi.org/10.1103/PhysRevB.83.235411

[44] Froudakis, G.E. (2001) Why Alkali-Metal-Doped Carbon Nanotubes Possess High Hydrogen Uptake. Nano Letters, 1, 531-533. http://dx.doi.org/10.1021/nl0155983

[45] Kubas, G.J. (2007) Fundamentals of $\mathrm{H}_{2}$ Binding and Reactivity on Transition Metals Underlying Hydrogenase Function and $\mathrm{H}_{2}$ Production and Storage. Chemical Reviews, 107, 4152-4205. http://dx.doi.org/10.1021/cr050197j 